\title{
Refinement of Protein NMR Structure under Membrane-like Environments with an Implicit Solvent Model
}

\author{
JunGoo Jee ${ }^{*}$ and Hee-Chul Ahn* \\ Center for Priority treas, Tokvo Metropolitan Lniversity, 1-1 Minami-Osaw'a, Hachioji, Tokvo 192-0373, Japan \\ E-mail: jee-jungooíatmu.ac.jp \\ Adwanced Analusis Center, Korea Institute of Science and Technologv, Sungbuk-gu, Seoul 136-791, Korea \\ Received February 18, 2009. Accepted April, 6, 2009
}

\begin{abstract}
Refinement of NMR structures by molecular dynamics (MD) simulations with a solvent model has improved the structural quality. In this study, we applied MD refinement with the generalized Bom (GB) implicit solvent model to protein structure determined under membrane-like environments. Despite popularity of the GB model, its applications to the refinement of NMR structures of hydrophobic proteins, in which detergents or organic solvents enclose proteins, are limited, and there is little infonnation on the use of another GB parameter for these cases. We carried out MD refinement of crambin NMR structure in dodecy lphosphocholine (DPC) micelles (Ahn el al., $/$. A $m$. Chem. Soc. 2006, 128,4398-4404) with GB/Surface area model and two different surface tension coefficients, one for aquatic and the other for hydrophobic conditions. Our data show that, of two structures by $M D$ refinement with $\mathrm{GB}$ model, the one refined with the parameter to consider hydrophobic condition had the better qualities in terms of precision and solvent accessibility.
\end{abstract}

Key Words: NMR, Implicit solvent. Molecular dy namics simulation. Generalized Born model, Force field

\section{Introduction}

Rapid adyances in calculation power and algorithms have made it practicable to employ molecular dynamics (MD) simulations with a sophisticated force field and a solvent model to refine NMR structures. However. the use of an explicit solvent model. in which solvent molecules such as water are added to a solute. is still demanding because of too large computing time to calculate an NMR ensemble comprising several ten stnictures. Instead. an implicit solvent model. where continuous media mimic the solvent's effects. has been successfully exploited. The generalized Born (GB) model, an implicit solvent model in most cases. has improved the qualities of NMR structures to levels similar to those achieved with explicit water refinement. ${ }^{.}$

$\mathrm{MD}$ refinement with the GB model is effective especially to confine the regions that do not converge only with NOE and torsion angle restraints by applying forces between atoms in the solute, and the solute and the solvent. For instance, the regions where hydrogen bonds and electrostatic interactions are dominant often suffer from a lack of NOE restraints, and MD refinement is helpful in such cases. The fast exchange feature of the weak protein complex also hinders gaining sufficient NOE restraints to confine the geometries of the interfaces. Our previous data showed that MD refinement with an AMBER force field and the GB model revealed better geometries of the interfaces in weak protein complexes. ${ }^{2.4}$ Furthermore, the GB model can be extended to find the correct 3D fold of proteins with only sparse NOE restraints by using the advanced sampling method, replica exchange.

Similar difficulties confront us in determining the membrane protein stnicture using solution NMR spectroscopy. Even in NMR samples that show well-dispersed backbone $2 \mathrm{D}\left[{ }^{15} \mathrm{~N}\right.$. $\left.{ }^{1} \mathrm{H}\right]$ spectra. the signals of the side-chains are often invisible or severely overlapped due to the inhomogeneity of the samples under membrane-like environments. Many side-chains lack NOE restraints on which NMR is heavily dependent for the calculation of structure. ${ }^{6}$ In several cases. extra long-range backbone restraints from paramagnetic relaxation enhancentent (PRE) and residual dipolar coupling (RDC) experiments have aided the improvement of NOE-based structures. ${ }^{~}{ }^{8}$ Compared with those of other protein NMR structures in solution, however, the qualities are still worse. and unrefined conformations of side-chains impede clear understanding of their roles.

We have been inspired to apply $\mathrm{MD}$ refinement with the GB model to calculating membrane protein structures. However. only a limited number of membrane protein structures have been solved by NMR (http:/Www.drorlist com/nmr/ MPNMR html). There is no report on refinement by the GB model. while there have been several $\mathrm{MD}$ simulation and folding studies of membrane proteins using the GB model. ${ }^{9.11}$ Before applying $\mathrm{MD}$ refinement to membrane proteins, it is necessary to know whether the GB model with default parameters for lyddroplulic conditions can intprove NMR structures under membrane-like environments or whether a new hydrophobic parameter is needed.

We chose crambin in dodecy lphosphocholine (DPC) micelles as a model protein. Crambin is a hydrophobic and waterinsoluble protein consisting of 46 residues. Organic solvents or detergents such as DPC are required to solubilize crambin. The NMR structure of crambin under DPC micelles (crambin $^{\mathrm{DFC}}$ ) was solved recently by multidimensional NMR experiments (PDB ID: 1YVA) ${ }^{11}$ The paramagnetic NMR experiment using $\mathrm{Mn}^{2+}$ showed that most of crambin's regions are surrounded by DPC micelles and thus are hydrophobic. except for small parts of the loop region. ${ }^{11}$ In this study. we carried out $\mathrm{MD}$ refinement of crambin NMR structure with $\mathrm{GB} /$ Surface area model and two different surface tension coefficients. 


\section{Experimentals}

We extracted the experimental distance and torsion angle restraints (1YVA.mr) from PDB database (http://nww. resb.org). The restraints consisted of $637 \mathrm{NOE} .76$ torsion angles and 38 lyydrogen bonds restraints. ${ }^{11}$ We first used CYANA (version $2.1)^{12}$ to generate 100 stnictures that satisfy experimental restraints. The torsion angle dynamics step in CYANA was 15,000. Then. we performed MD refinement with experimental NMR restraints. and an all-atom force field and the $G B$ model. We used AMBER force field (ff03) and AMBER 9 software package ${ }^{13.14}$ This $\mathrm{MD}$ refinement consisted of three stages: energy minimization with 1500 steps. molecular dynamics of $20 \mathrm{ps}$. and energy minimization with 1500 steps. ${ }^{2--}$ Among the 100 stnictures. 20 structures showing both the lowest AMBER energies and no significant violation in distance $(<0.3 \AA)$ and torsion angle $\left(<3^{\circ}\right)$ restraint were selected as the final ensemble. For the experimental distance and torsion angle restraints. we used the force constants of 50 $\mathrm{kcal} \cdot \mathrm{mol}^{-1} \cdot \mathrm{A}^{-2}$ and $200 \mathrm{kcal} \cdot \mathrm{mol}^{-1} \cdot \mathrm{rad}^{-2}$, respectively.

\section{Results and Discussion}

The GB model forcunent study. AMBER 9 can implement $6 \mathrm{~GB}$ models $(i g b=1,2.5,6,7.10)$. For the GB model in the current study, we selected the GB/Surface Area (GB/SA. igb $=2 . \mathrm{GB}^{\mathrm{BB}}$ ) model.$^{15}$ Detailed explanation of all the $\mathrm{GB}$ models is beyond the scope of this study. We briefly describe the basic idea employed for the current study. In the GB/SA model, the free energy of solvation. $J G_{\text {s. } \mathrm{l}}$. can be decomposed further into polar. $J G_{\text {polar. }}$ and non-polar. $J G_{\text {nonpolar. }}$ contributions as $\left.J G_{\text {solv }}=\right\rfloor G_{\text {polar }}+J G_{\text {nonpolar }}$. The $J G_{\text {polar }}$ term is based on the generalized Born formula derived from the Poisson-Boltzmann equation. The $\mathcal{J} G_{\text {tronpolar }}$ term is the product of the surface tension coefficient (surften. $\mathrm{kcal} \cdot \mathrm{mol}^{-1} \cdot \mathrm{A}^{-2}$ ) and the surface area $\left(S t, \hat{A}^{2}\right)$. and is expressed as $j G_{\text {nonpolar }}=$ surften $* S A$. Therefore. by increasing the value of surften. we can increase the contribution of the non-polar term. By setting the values of sturfen to 0.005 and 0.04 , we repeated $\mathrm{MD}$ refinement with the same experimental restraints. The default value of surften in AMBER 9 was 0.005 for the aquatic condition. and the value of 0.04 was adapted from the folding study of the membrane protein using the GB model. ${ }^{16}$

$\mathrm{MD}$ refinement improved the qualities of crambin structures. Table 1 shows the statistics of the final structures in

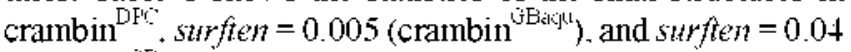
(crambin ${ }^{\text {(Einterl) }}$ ). The refined structures did not show a large deviation from the reference ones. The pairwise differences in the backbone RMSD values between crambin ${ }^{\mathrm{DPC}}$ and crambin ${ }^{\text {GEaqu }}$. and crambin ${ }^{\mathrm{DPC}}$ and crambin ${ }^{\text {GEmem }}$ were $1.26 \pm$ $0.26 \AA$ and $1.26 \pm 0.28 \AA$. respectively: Crambin ${ }^{2 B a q u}$ and crambin ${ }^{\text {GETem }}$ were very similar to each other. showing pairwise RMSD values of $0.90 \pm 0.28 \AA$. Both structures by $\mathrm{MD}$ refinement exhibited improvements in precision and accuracy. The mean RMSD values of the backbone (heavy) atoms in residues $1-46$ improved from $0.70 \pm 0.11 \AA(1.02 \pm$ $0.11 \AA$ ) for crambin ${ }^{\mathrm{DPC} C}$ to $0.61 \pm 0.19 \AA(0.88 \pm 0.17 \AA)$ for

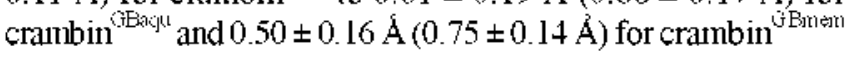
(Table 1). Further, the most favored region in the Ramachandran analy sis improved from $79.6 \%$ to $91.0 \%$ and $89.7 \%$ for

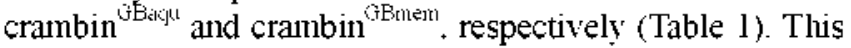
result demonstrates the robustness of including MD refinement for NMR structure calculations and is consistent with our previous data for water-soluble proteins. ${ }^{2-4}$

It is noticeable that the structures by $\mathrm{MD}$ with the GB model in the current study outdid those by the most popular NMR refinement protocols using DMSO (PDB ID: 2EYC) and water (PDB ID: 2EYD) explicit solvents of CNS/ARIA ${ }^{1 \text { ? }}$ in terms of qualities. The mean backbone RMSD values in the residues $1-46$ were 0.89 and 0.94 by DMSO and water-refined crambin structures. respectively. ${ }^{11}$ We reason that this refinement method with explicit solvents does not use soplisticated protocols in the cut-off value for non-bond interactions and the $\mathrm{MD}$ schedule due to the compromise between performance and CPU efficiency.

Residue-specific solvent accessible surface areas revealed the differences in each structure. To obtain detailed insight into the differences in these structures. we calculated the

Table 1. Statistics of crambin ${ }^{\mathrm{DPC}}$, crambin ${ }^{\mathrm{GEaqu}}$, and crambin ${ }^{\mathrm{CB} m e m}$

\begin{tabular}{|c|c|c|c|}
\hline & crambin $^{\mathrm{DPC}}$ & 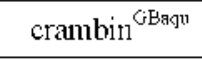 & crambin $^{\mathrm{GBmem}}$ \\
\hline \multicolumn{4}{|l|}{ Energy $(\mathrm{kcal} / \mathrm{mol})$} \\
\hline AMBER GB energ: & Not & $-1068 \pm 3$ & $-967 \pm 4$ \\
\hline Constraints energy & available & $14 \pm 2$ & $14 \pm 1$ \\
\hline \multicolumn{4}{|l|}{ Maximunn Violation } \\
\hline Distance violation $(\AA)$ & 0.430 & 0.265 & 0.252 \\
\hline Angle violation (degree) & 0.94 & 0.0 & 0.0 \\
\hline \multicolumn{4}{|l|}{ Mean RMSD $(A)$} \\
\hline Backbone & $0.70 \pm 0.11$ & $0.61 \pm 0.19$ & $0.50 \pm 0.16$ \\
\hline Heavy atoms & $1.02 \pm 0.11$ & $0.88 \pm 0.17$ & $0.75 \pm 0.14$ \\
\hline \multicolumn{4}{|l|}{ Ranachandran (\%) } \\
\hline Most favored region & 79.6 & 91.1 & 90.0 \\
\hline Additionally allowed region & 17.7 & 8.9 & 10.0 \\
\hline Generously allowed region & 2.0 & 0.0 & 0.0 \\
\hline Disallowed region & 0.7 & 0.0 & 0.0 \\
\hline
\end{tabular}



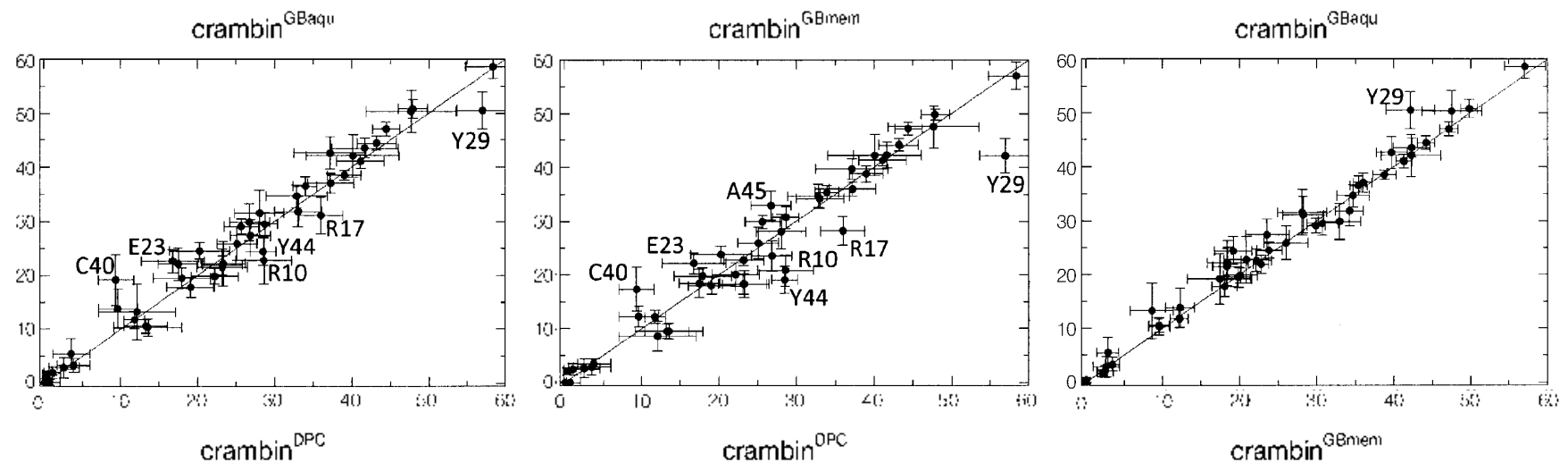

Figure 1. Pairwise comparisons of residue-specific solvent accessible sufface area (RASA) values among crambin ${ }^{\text {In' }}$ (PDB ID: IYVA),

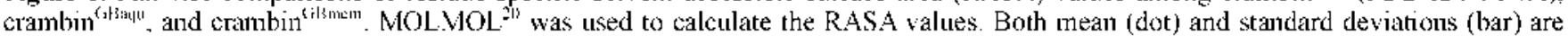
shown. Labels for the $x$-axes are witten at the bottoms of the figures, whereas the labels for the $y$-axes are drawn at the top. The " $y=x "$ line is also drawn as a reference. Significant outliers are labeled. The unit of each axis is percentage $(\%)$.
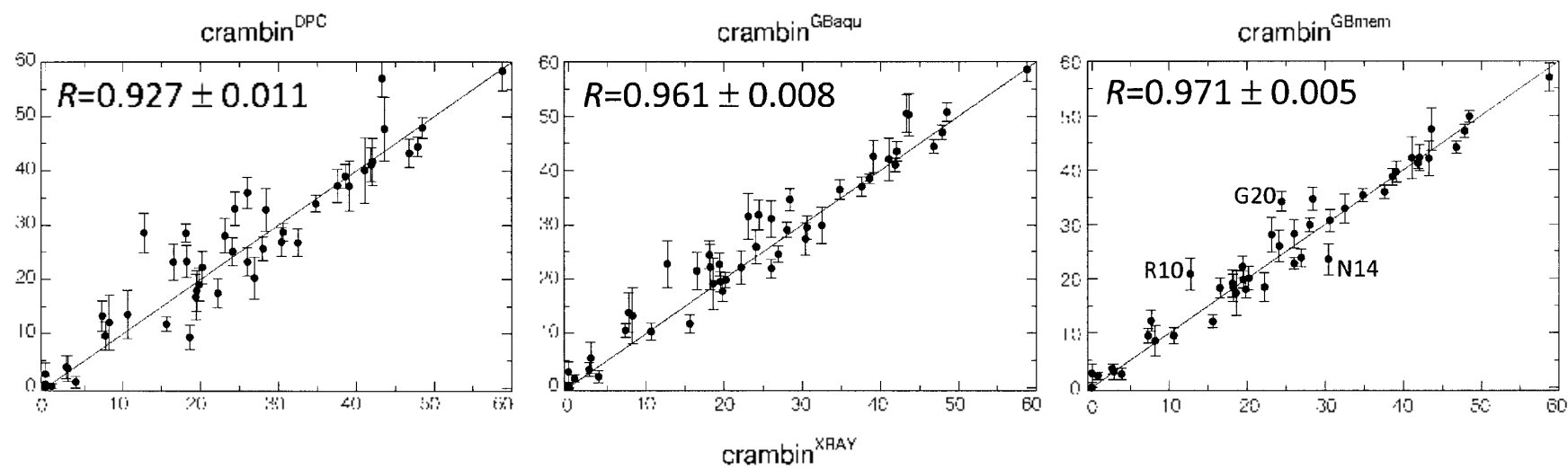

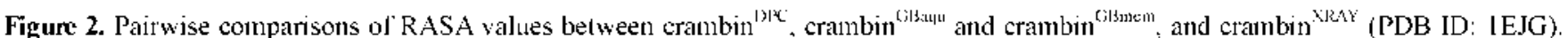
Figures were generated with the same notations as Figure 1. The Pearson correlation coefficient $(K)$ values were calculated with the MATLAB package (Mallwworks Inc., MA) wilh 1000 Monte Carlo cycles. For clarity, only outliers in crambin! ${ }^{\text {Gijsmem }}$ are shown

residue-specific solvent accessible surface area (RASA). If solvents can contact a residue freely, the RASA value is $100 \%$. In contrast, if a residue is perfectly blocked from solvents, the RASA value is $0 \%$. Therefore, RASA values are good indices to know the effects of an implicit solvent in the refinement. Pairwise comparisons of the RASA values among

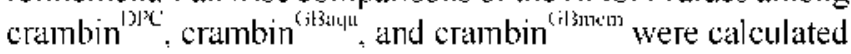
(Figure 1). As predicted from the statistics (Table 1), there was a small difference in the pairwise RASA (pRASA) value

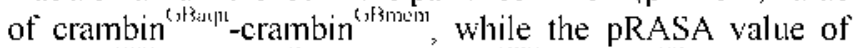

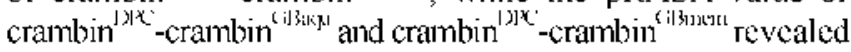
several outliers. H lowever, it should be noted that there was a tendency for the RASA values of crambin ${ }^{\text {il|kenlu }}$ to be slightly greater than those of crambin ${ }^{\text {iikturin, }}$, although the difference was not large (Figure 1). This implics that crambin ${ }^{\text {ifisinl }}$ favored solvents more, and shows the role of surften tem in the GBSA model. Among the outliers in the pRASA values between crambin ${ }^{\text {IMx: }}$ and the structures refined by the GB model, the most distinct one was Ty -29 because it is a part of the $\alpha$-helix and structural changes due to MD refinement are usually not large in the $\alpha$-helices and $\beta$-sheets. Tyr- 29 was also the sole outlier in the pRASA of crambin ${ }^{\text {(ilkiklu }}$-crambin ${ }^{\text {(il) } 3 m e m}$. Intriguingly, Tyr- 29 was one of the residues that showed the largest chemical shift change in the $2 \mathrm{D}\left[{ }^{15} \mathrm{~N},{ }^{1} \mathrm{H}\right] \mathrm{HSQC}$ of crambin in DPC and dihexanoyl phosphatidy lcholine (DHPC) micelles:" this implies that it was sensitive to environmental conditions even in actual situations. On the other hand, other big outliers, namcly, $\mathrm{Arg}-17$, Cys-40, and Ty $\mathrm{r}-44$, arc located in bent or lcop regions, where conformations change occasionally due to MD refinement.

Residue-specific solvent accessible sufface areas between crambin $^{\mathrm{CBnnm}}$ and crambin ${ }^{\mathrm{XRAY}}$ were in better aygrement. We further compared the RASA values of these structures with that of the $X$-ray crambin structure (PDI ID: 1 IJJG, crambin $^{X \times A Y}$ ), which was crystallized under an ethanol and water mixture solution. ${ }^{18}$ Crambin ${ }^{X R A Y}$ is a fine structure with the best resolution $(0.54 \AA)$ among the available crambin structures. The RASA values of crambin ${ }^{\text {tifmem }}$ showed the best Pearson correlation coefficient $(R)$ value of $0.971 \pm 0.005$ over crambin ${ }^{X R A Y}$,

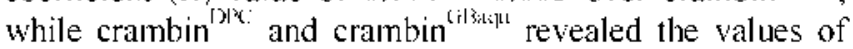
$0.927=0.011$ and $0.961=0.008$, respecilvely (Figure 2 ). In particular, most of the outliers that were found in the pRASA values of crambin ${ }^{\text {IPC: }}$-crambin ${ }^{\text {(ilimom }}$ disappeared in that of

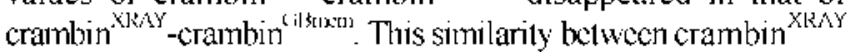
and crambin ${ }^{\text {filbmem }}$ can also be visualized by inspecting the side-chain orientations of aromatic residues. The orientations 


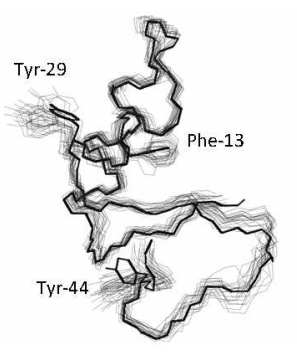

crambin $^{\mathrm{DPC}}$

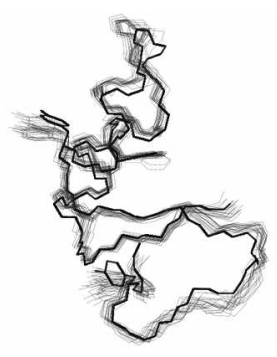

crambin ${ }^{\text {GBacu }}$

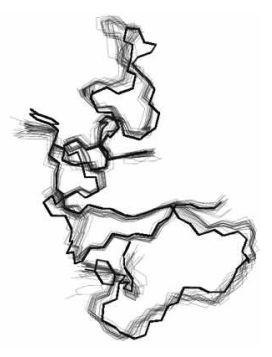

crambin $^{\text {GEnien }}$
Figure 3. Overlay figures of crambin ${ }^{\mathrm{DPC}}$, crambin ${ }^{\mathrm{GBaqu}}$, and crambin ${ }^{\text {GBmem }}$ against crambin ${ }^{2} 4{ }^{\prime}$. MOLMOL was used to generate these figures. Crambun ${ }^{\text {RAY }}$ is presented with bold lines and the others with nanow lunes. Side-chans of aromatic residues, Phe-13, Tyr-29, and Tyr-44, are also shown.

Table 2. $\chi_{4}$ angle statistics of aromatic residues

\begin{tabular}{|c|c|c|c|c|}
\hline & crambin $\mathrm{XR} A 3$ & crambin $^{\text {DPI }}$ & crambont $^{\text {Gaqu }}$ & crambin $^{\text {GBrrem }}$ \\
\hline Phe-1 3 & 179.6 & $154.6 \pm 12.7$ & $173.5 \pm 6.7$ & $171.4 \pm 6.8$ \\
\hline Tyr-29 & 161.3 & $-179.5 \pm 24.2$ & $168.0 \pm 6.1$ & $152.9 \pm 5.5$ \\
\hline Tyr-4t & -69.5 & $147.6 \pm 115.0$ & $-48.5 \pm 3.1$ & $-53.2 \pm 3.6$ \\
\hline
\end{tabular}

of the side-chains in all the aromatic residues (Phe-13. Ty r-29. and $\mathrm{Tyr}-4+4)$ converged well in both the GB-model-based structures compared with those in crambin ${ }^{\mathrm{DPC}}$ (Figure 3). The

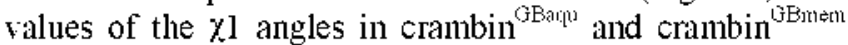
were consistent with those in crambin ${ }^{\mathrm{KRAY}}$, whereas the rotation of Tyr-4t was totally different and those in Phe-13 and Tyr-29 were more deviated in crambin ${ }^{\mathrm{DPC}}$ (Table 2). One may argue that detailed analysis is less meaningful because the solvents in crambin ${ }^{\mathrm{KRAY}}$ are different from those in the current study. However. the purpose of comparison is to gain insight into the conformations of side-chains that are mainly influenced by solvents. and our analysis focused on the side-chains in a qualitative way.

For the general application of the GB model to proteins under membrane-like environments. One may criticize that crambin is a special case and most membrane proteins contain both hydrophobic and hydrophilic parts and that we cannot define the system with a global parameter. surfien. However. recent developments in the GB model have enabled to describe a system into several layers of different surface tensions. Actually. it has been successfully applied to folding and several MD studies of membrane proteins. ${ }^{9.1(1}$ We can apply the same algorithm to the NMR stnicture determination of real membrane proteins, as long as the boundaries of the membrane and aquatic parts are identified by experiments.

\section{Conclusion}

Our data, first. showed that MD refinement with an AMBER force field and the GB model improved the accuracy and precision of NMR structures. Second. the GB model with a parameter considering the membrane-like condition yielded more improvements than those with the nonnal GB parameter. We do not ignore the imperfectness of current GB model. ${ }^{15}$ However. it should be again noted that both crambin ${ }^{\mathrm{GEmem}}$ and crambin ${ }^{\text {Bicksu }}$ did not show any violation of the experimental restraints. Different from folding study, the conformational spaces to search in NMR stnucture refinement are narrow because of the experimental restraints. and the errors due to GB model will be much small. Our data will be an initial step toward structure determination of membrane proteins using NMR data.

Aclonowledgments. JGJ appreciates Prof. Masahiro Shirakawa for his giving the initial motivation to study MD refinements.

\section{Refelences}

1. Xia, B.; Tsui, V.; Case, D. A.; Dyson, H. J.; Wright, P. E. J. Biomol. MRR 2002, 22,317-331.

2. Tee, J.; Byeon, I. T.; Louis, T. M: Gronenbon, A. M. Proteins $2008,71,1420-1431$.

3. Ohno, A.: Jee, T; Fujiwara, K.; Tenno, T.: Goda, N.; Tochio, H.; Kobayashi, H.; Hiroaki, H.; Shirakawa, M. Structure 2005, 13, $521-532$.

4. Fujiwara, K.: Tenno, T.: Sugasawa, K.; Jee, J. G.; Olki, I.; Kojima, C; Tochio, H.; Hroak, H.; Hanaoka, F.; Shırakawa, M. J. Biol. Chem. 2004, 279, 4760-4767.

5. Chen, T. Im, W. Brooks, C. L., 3rd J. Ant. Chent. Soc. 2004, 126, 16038-16047.

6. Wuthrich, K. MAR of Proteins and Nicleic Acids; Wiley: New York, 1986

7. Call, M. E; Schnell, I. R.; Xu, C; Lut, R. A; Chou, J. T.; Wucherptennig, K. W. Cell 2006, 127, 355-368.

8. Roosild, T. P.; Greenwald, J.; Vega, M.; Castronovo, S.; Riek, R.; Choe, S. Science 2005, 307, 1317-1321.

9. Feig, M. Methods Mol Biol 2008, \$43, 181-196.

10. Chen, J.; Brooks, C. L., 3rd, Khandogm, T. Cum Opin. Struct. Biol. 2008, 18, 140-148.

11. Ahn, H. C.; Turanic, N.; Macura, S.; Markley, I. L. J. Am. Chem. Soc. 2006, $128,4398-4404$.

12. Guntert, P, Mumenthaler, C.; Wuthrich, K. J. Mol Biol 1997, $273,283-298$.

13. Case, D. A.; Cheatham, T. E., 3rd; Darden, T: Gohlke, H.; Luo, R.; Mer, K. M., Ir.; Onufnev, A.: Smmerling, C.: Wang, B.; Woods, R. J. J. Comput. Chem. 2005, 26, 1668-1688.

14. Ponder, J. W: Case, D. A. Adv Protein Chem. 2003, 66, 27-85.

15. Onutriev, A.: Bashford, D.: Case, D. A. Proteins 2004, 55 , 383-394

16. Im, W; Brooks, C. L., 3rd Proc. Natl. Acad. Sci. LS.4 2005, 102, 6771-6776.

17. Linge, T. P.; Williams, M. A.; Spronk, C. A.; Bonvin, A. M.; Nilges, M. Proteins 2003, 50, $496-506$

18. Jelsch, C.: Teeter, M. M.: Lamzin, V: Pichon-Pesme, V:; Blessing, R. H.; Lecomte, C. Proc. Natl Acad. Sci. LS.4 2000, 97.3171-3176.

19. Chen, J.: Brooks, C. L., 3rd Phss. Chem. Chem Phys. 2008, 10 , 471-481.

20. Koradi, R.; Billeter, M.; Wuthrich, K. J. Hol Givaph 1996, It, $51-55.29-32$ 